\title{
The Effects of Glucose Lowering Agents on the Secondary Prevention of Coronary Artery Disease in Patients with
} Type 2 Diabetes

\author{
Inha Jung ${ }^{1}$, Hyemi Kwon ${ }^{1}$, Se Eun Park ${ }^{1}$, Kyung-Do Han², Yong-Gyu Park ${ }^{3}$, Eun-Jung Rhee ${ }^{1}$, Won-Young Lee ${ }^{1}$ \\ ${ }^{1}$ Division of Endocrinology and Metabolism, Department of Internal Medicine, Kangbuk Samsung Hospital, Sungkyunkwan \\ University School of Medicine; ${ }^{2}$ Department of Statistics and Actuarial Science, Soongsil University; ${ }^{3}$ Department of \\ Biostatistics, Biomedicine \& Health Sciences, College of Medicine, The Catholic University of Korea, Seoul, Korea
}

Background: Patients with diabetes have a higher risk of requiring repeated percutaneous coronary intervention (PCI) than non-diabetic patients. We aimed to evaluate and compare the effects of anti-diabetic drugs on the secondary prevention of myocardial infarction among type 2 diabetes mellitus patients.

Methods: We analyzed the general health check-up dataset and claims data of the Korean National Health Insurance Service of 199,714 participants (age $\geq 30$ years) who underwent PCIs between 2010 and 2013. Those who underwent additional PCI within 1 year of their first PCI $(n=3,325)$ and those who died within 1 year $(n=1,312)$ were excluded. Patients were classified according to their prescription records for glucose-lowering agents. The primary endpoint was the incidence rate of coronary revascularization.

Results: A total of 35,348 patients were included in the study. Metformin significantly decreased the risk of requiring repeat PCI in all patients (adjusted hazard ratio [aHR], 0.77). In obese patients with body mass index (BMI) $\geq 25 \mathrm{~kg} / \mathrm{m}^{2}$, patients treated with thiazolidinedione (TZD) exhibited a decreased risk of requiring repeat revascularization than those who were not treated with TZD (aHR, 0.77; 95\% confidence interval, 0.63 to 0.95 ). Patients treated with metformin showed a decreased risk of requiring revascularization regardless of their BMI. Insulin, meglitinide, and alpha-glucosidase inhibitor were associated with increased risk of repeated PCI.

Conclusion: The risk of requiring repeat revascularization was lower in diabetic patients treated with metformin and in obese patients treated with TZD. These results suggest that physicians should choose appropriate glucose-lowering agents for the secondary prevention of coronary artery disease.

Keywords: Diabetes mellitus; Coronary artery disease; Secondary prevention; Percutaneous coronary intervention

Received: 26 March 2021, Revised: 27 July 2021, Accepted: 6 August 2021

Corresponding authors: Eun-Jung Rhee

Division of Endocrinology and Metabolism, Department of Internal Medicine, Kangbuk Samsung Hospital, Sungkyunkwan University School of Medicine, 29 Saemunan-ro, Jongno-gu, Seoul 03181, Korea

Tel: +82-2-2001-2485, Fax: +82-2-2001-2049, E-mail: hongsiri@hanmail.net

Won-Young Lee

Division of Endocrinology and Metabolism, Department of Internal Medicine, Kangbuk Samsung Hospital, Sungkyunkwan University School of Medicine, 29 Saemunan-ro, Jongno-gu, Seoul 03181, Korea

Tel: +82-2-2001-2579, Fax: +82-2-2001-2049, E-mail: drlwy@hanmail.net

\section{Copyright $\odot 2021$ Korean Endocrine Society}

This is an Open Access article distributed under the terms of the Creative Commons Attribution Non-Commercial License (https://creativecommons.org/ licenses/by-nc/4.0/) which permits unrestricted non-commercial use, distribution, and reproduction in any medium, provided the original work is properly cited. 


\section{INTRODUCTION}

Cardiovascular (CV) morbidity in patients with type 2 diabetes mellitus (T2DM) is a major concern in clinical practice. Patients with T2DM are at an increased risk of coronary artery disease (CAD) and CV mortality compared to patients without diabetes $[1,2]$. Percutaneous coronary intervention (PCI) is an essential treatment modality for CAD. Although drug-eluting stents (DESs) have been widely used to inhibit vascular proliferation, the rates of restenosis and mortality in T2DM patients who have undergone PCI are still higher than those in non-diabetics [3]. Several meta-analyses have reported a significant increase in the risk of myocardial infarction (MI) or sudden death among patients taking rosiglitazone. The Food and Drug Administration (FDA) has provided guidance for the pharmaceutical industry, which requires the assessment of $\mathrm{CV}$ outcomes of all new antidiabetic drugs [4]. A number of cardiovascular outcome trials (CVOTs) have been conducted since the issuance of the FDA guidance. In most CVOTs, a new antidiabetic drug is added to the standard of care treatments in patients with high CV risk, and $\mathrm{CV}$ outcomes were compared with the standard of care treatment alone. The majority of CVOTs used major adverse cardiovascular events (MACEs), including nonfatal MI, nonfatal stroke, and $\mathrm{CV}$ death as the primary endpoint to demonstrate $\mathrm{CV}$ safety.

To date, findings from these trials suggest beneficial class effects of sodium-glucose cotransporter-2 (SGLT-2) inhibitors and glucagon-like peptide-1 receptor agonists (GLP-1RAs) in reducing primary $\mathrm{CV}$ events and secondary prevention [5]. Although newer glucose-lowering agents such as SGLT-2 inhibitors and GLP-1RAs have demonstrated cardioprotective properties, clinicians in the real world are reluctant to prescribe these drugs. A significant number of patients want "old" drugs instead of new antidiabetic drugs because of economic burdens or because GLP-1RAs require subcutaneous injection. Many older antidiabetic drugs do not have published CVOTs; however, some have published CV outcomes. Clinical trials with dipeptidyl peptidase-4 inhibitors (DPP-4is) have suggested a neutral effect on major CV events in patients with T2DM and established cardiovascular disease (CVD), and reported a higher risk of requiring hospitalization for heart failure in the saxagliptin treatment group [6,7]. Regarding thiazolidinedione (TZD), the PROactive study suggested a favorable effect of TZD on the secondary prevention of MACE [8]. However, a meta-analysis highlighted substantial uncertainty regarding the CV safety of TZDs [9].

As mentioned above, the cardioprotective effects of different glucose-lowering agents remain controversial. Moreover, realworld data suggesting the effects of each glucose-lowering agent on the prevention of repeat revascularization, especially among patients who underwent PCI, are scarce. Therefore, we evaluated the rate of coronary revascularization as the primary outcome. Using claims data and health examination data from the National Health Insurance Service (NHIS) in Korea, we evaluated and compared the effects of different glucose-lowering agents on secondary prevention of revascularization among patients with T2DM and documented CAD.

\section{METHODS}

\section{Source of data}

This study analyzed data from the Korean NHIS and claims databases. The NHIS is a mandatory health insurance program that covers $97.1 \%$ of the Korean population. In Korea, the NHIS is a single-payer program managed by the government. The NHIS includes an eligibility database (comprising data on age, sex, socioeconomic variables, type of eligibility, and household income level), a medical treatment claims database (based on medical bills that were claimed by medical service providers for their medical expenses), a health examination database (results of general health examinations and questionnaires on lifestyle and behavior), a medical care institution database (types of medical care institutions, location, equipment, and number of physicians), and death register. We used the general health examination data and NHIS claims data, including data pertaining to diagnoses, procedures, prescription records, and mortality. This study was approved by the Institutional Review Board of Kangbuk Samsung Hospital of Korea (KBSMC 2018-07-024). Due to a retrospective nature of our study and anonymous information we used, informed consent was waived by the board. All personal information was deleted, and only non-identifiable data were used for the analyses.

\section{Study population and design}

This study investigated adults with a documented history of PCI, aged $\geq 30$ years, and who underwent a general health check-up program at least twice. We selected 199,714 participants who underwent PCI between 2010 and 2013. We only included participants who had undergone a health check-up within 1 year from the date of their PCI. We excluded patients who had repeat revascularization $(n=3,325)$ or died $(n=1,104)$ within 1 year after prior PCI. We excluded 3,325 participants to ensure that their repeat PCI is not a part of a staged PCI. A diagnosis of 
T2DM was defined according to the following criteria: (1) the presence of International Classification of Diseases, 10th Revision, Clinical Modification (ICD-10-CM) codes E11, E12, E13, or E14 and claims for at least one oral antidiabetic agent or insulin at baseline [10], or (2) fasting glucose level $\geq 126 \mathrm{mg} / \mathrm{dL}$ (obtained from the health examination database). Subjects with missing data were excluded from the study. Finally, 35,348 participants were included in the analysis. The incidence of repeat PCI in the claims database was analyzed from January 1, 2010, to December 31, 2017, or until the date of death, whichever came first (Supplemental Fig. S1).

\section{Anthropometric and laboratory measurement}

Data on medical history and health-related behaviors were collected through a self-reported questionnaire, whereas physical measurements and serum biochemical parameters were obtained by trained staff. Body weight was measured in light clothing with no shoes to the nearest $0.1 \mathrm{~kg}$ using a digital scale. Height was measured to the nearest $0.1 \mathrm{~cm}$. Systolic and diastolic blood pressures were measured using a standardized sphygmomanometer. Body mass index was defined as the patients' weight $(\mathrm{kg})$ divided by the square of the height $(\mathrm{m})$. Fasting blood glucose, aspartate aminotransferase, alanine aminotransferase, and total cholesterol levels were measured after 12 hours of fasting.

\section{Definition of comorbid disease}

Hypertension was defined according to the presence of at least one claim per year for the prescription of antihypertensive agents, under ICD-10-CM codes I10-I15, or a systolic/diastolic $\mathrm{BP} \geq 140 / 90 \mathrm{~mm} \mathrm{Hg}$. The presence of dyslipidemia was defined according to the presence of at least one claim per year for the prescription of antihyperlipidemic agents under ICD-10 codes E78 or total cholesterol $\geq 240 \mathrm{mg} / \mathrm{dL}$.

\section{Classification of glucose-lowering agents}

The glucose-lowering agents in this study were grouped into seven classes: insulin, sulfonylurea (SU), metformin, meglitinide, TZD, DPP-4 inhibitors (DPP-4i), and alpha-glucosidase inhibitors (AGI). We obtained information on prescriptions (i.e., the class of the drug, date prescribed, days of supply, and quantity dispensed). Participants who took more than two different classes of antidiabetic drugs were considered to be undergoing combination therapy [11]. We classified therapies as monotherapy, dual therapy, and triple therapy.

\section{Primary outcome}

The primary outcome was repeat revascularization with PCI during the follow-up period. PCI was defined using the following codes: NHIS M6551-6552, 6561-6564, and 6571-6572.

\section{Statistical analysis}

Continuous variables are presented as mean \pm standard deviation, and categorical variables are expressed as percentages. The clinical characteristics of the participants were compared using one-way analysis of variance for continuous variables and the chi-square test for categorical variables. The incidence rate of repeat PCI is presented per 1,000 person-years. Cox proportional hazards regression analysis was used to calculate the hazard ratio (HR) and 95\% confidence intervals (CIs) for repeat PCI according to the individual glucose-lowering agents. Adjusted HRs were calculated by adjusting the following variables: age, sex, household income, hypertension, dyslipidemia, smoking, alcohol consumption, exercise frequency, body mass index (BMI), use of insulin, and the number of oral antidiabetic agents. For subgroup analysis, we stratified the participants according to their BMI.

All reported $P$ values were two-tailed, and $P$ values $<0.05$ were considered statistically significant. All statistical analyses were performed using SAS version 9.3 (SAS Institute Inc., Cary, NC, USA) and R version 3.2.3 (The R Foundation for Statistical Computing, Vienna, Austria, http://www.Rproject.org).

\section{RESULTS}

\section{Baseline characteristics of participants}

The mean age of the participants was $64.6 \pm 9.52$ years and $23,991(67.9 \%)$ of the participants were men. The incidence of repeat PCI during a mean follow-up period of $4.27 \pm 1.73$ years was analyzed. Table 1 presents the participants' baseline characteristics. The proportion of men was higher in patients who underwent repeat revascularization with PCI than in those who did not. Compared with subjects who did not undergo repeat PCI, those who underwent repeat PCI had a higher rate of insulin treatment $(P<0.001)$ (Table 1). Participants who underwent repeat PCI had a higher BMI and were more obese. Additionally, those who did not undergo repeat PCI had lower fasting blood glucose and triglyceride levels than those who underwent repeat PCI.

\section{Risk of requiring repeat $\mathrm{PCI}$ according to individual glucose lowering agents}

We compared the effects of anti-diabetic drugs on reducing the 
Table 1. Baseline Characteristics of Study Participants

\begin{tabular}{|c|c|c|c|c|}
\hline \multirow{2}{*}{ Characteristic } & \multirow{2}{*}{ Total } & \multicolumn{3}{|c|}{ Repeat PCI } \\
\hline & & No & Yes & $P$ value \\
\hline Number & 35,348 & 30,903 & 4,445 & \\
\hline Age, yr & $64.6 \pm 9.52$ & $64.7 \pm 9.56$ & $64.0 \pm 9.22$ & $<0.0001$ \\
\hline Male sex & $23,991(67.9)$ & $20,900(67.6)$ & $3,091(69.5)$ & 0.0109 \\
\hline Household income, low $20 \%$ & $7,580(21.4)$ & $6,570(21.3)$ & $1,010(22.7)$ & 0.0264 \\
\hline Smoking status & & & & 0.2052 \\
\hline Never smoker & $18,113(51.2)$ & $15,853(51.3)$ & $2,260(50.8)$ & \\
\hline Former smoker & $9,580(27.1)$ & $8,329(27.0)$ & $1,251(28.1)$ & \\
\hline Current smoker & $7,655(21.7)$ & $6,721(21.8)$ & $934(21.0)$ & \\
\hline Alcohol drinking & & & & 0.2101 \\
\hline None & $25,171(71.2)$ & $21,961(71.1)$ & $3,210(72.2)$ & \\
\hline Mild (<30 g/day) & $8,380(23.7)$ & $7,373(23.9)$ & $1,007(22.7)$ & \\
\hline Heavy ( $\geq 30$ g/day) & $1,797(5.1)$ & $1,569(5.1)$ & $228(5.1)$ & \\
\hline Regular exercise & $7,578(21.4)$ & $6,665(21.6)$ & $913(20.5)$ & 0.1186 \\
\hline Use of insulin & $14,444(40.9)$ & $12,439(40.3)$ & $2,005(45.1)$ & $<0.0001$ \\
\hline Sulfonylurea & $21,006(59.4)$ & $18,261(59.1)$ & $2,745(61.8)$ & 0.0007 \\
\hline Metformin & $26,891(76.1)$ & $23,577(76.3)$ & $3,314(74.6)$ & 0.0111 \\
\hline Meglitinide & $1,626(4.6)$ & $1,356(4.4)$ & $270(6.1)$ & $<0.0001$ \\
\hline TZD & $1,796(5.1)$ & $1,583(5.1)$ & $213(4.8)$ & 0.3480 \\
\hline DPP4 inhibitor & $12,499(35.4)$ & $10,978(35.5)$ & $1,521(34.2)$ & 0.0886 \\
\hline Alpha-glucosidase inhibitor & $5,497(15.6)$ & $4,642(15.0)$ & $855(19.2)$ & $<0.0001$ \\
\hline No. of antidiabetic agents & & & & 0.0633 \\
\hline 0 & $4,723(13.4)$ & $4,161(13.5)$ & $562(12.6)$ & \\
\hline 1 & $6,738(19.1)$ & $5,922(19.2)$ & $816(18.4)$ & \\
\hline 2 & $11,919(33.7)$ & $10,429(33.8)$ & $1,490(33.5)$ & \\
\hline$\geq 3$ & $11,968(33.9)$ & $10,391(33.6)$ & $1,577(35.5)$ & \\
\hline Hypertension & $31,579(89.3)$ & $27,552(89.2)$ & $4,027(90.6)$ & 0.0036 \\
\hline Dyslipidemia & $31,932(90.3)$ & $27,953(90.5)$ & $3,979(89.5)$ & 0.0479 \\
\hline Chronic kidney disease & $7,771(22.0)$ & $6,658(21.5)$ & $1,113(25.0)$ & $<0.0001$ \\
\hline BMI, $\mathrm{kg} / \mathrm{m}^{2}$ & $25.0 \pm 3.06$ & $25.0 \pm 3.05$ & $25.1 \pm 3.07$ & 0.0374 \\
\hline SBP, $\mathrm{mm} \mathrm{Hg}$ & $129.5 \pm 16.59$ & $129.4 \pm 16.56$ & $130.0 \pm 16.83$ & 0.0177 \\
\hline DBP, mm Hg & $77.4 \pm 10.43$ & $77.4 \pm 10.42$ & $77.2 \pm 10.53$ & 0.2120 \\
\hline Total cholesterol, mg/dL & $175.4 \pm 47.2$ & $175.2 \pm 47.15$ & $176.8 \pm 47.49$ & 0.0405 \\
\hline Fasting blood glucose, mg/dL & $138.7 \pm 45.86$ & $138.3 \pm 45.42$ & $141.3 \pm 48.74$ & $<0.0001$ \\
\hline Waist circumference, $\mathrm{cm}$ & $86.7 \pm 8.06$ & $86.6 \pm 8.05$ & $87.0 \pm 8.07$ & 0.0009 \\
\hline Weight, kg & $65.7 \pm 10.87$ & $65.7 \pm 10.89$ & $66.2 \pm 10.7$ & 0.0057 \\
\hline LDL-C, mg/dL & $97.6 \pm 43.79$ & $97.4 \pm 43.81$ & $98.6 \pm 43.64$ & 0.0827 \\
\hline $\mathrm{TG}, \mathrm{mg} / \mathrm{dL}$ & $138.8(138.1-139.6)$ & $138.5(137.7-139.3)$ & $141.2(139.0-143.4)$ & 0.0210 \\
\hline
\end{tabular}

Values are expressed as mean \pm standard deviation, number $(\%)$, or median (interquartile range).

PCI, percutaneous coronary intervention; TZD, thiazolidinedione; DPP4, dipeptidyl peptidase-4; BMI, body mass index; SBP, systolic blood pressure; DBP, diastolic blood pressure; LDL-C, low density lipoprotein cholesterol; TG, triglyceride. 
risk of requiring repeat PCI among patients with T2DM. Metformin and DPP-4i significantly decreased the risk of requiring repeat PCI in the total study population. After adjusting for confounding variables such as insulin use and number of oral antidiabetic drugs, the hazard ratios (HRs) were attenuated but showed consistently reduced risks for repeat PCI among patients treated with metformin or DPP-4i (adjusted hazard ratio [aHR], 0.74 and 0.93, respectively; $P<0.001$ ) (Table 2, model $3)$. In the final model adjusted for fasting blood glucose and duration of diabetes, we found no statistical significance except for patients treated with metformin (aHR, 0.77; 95\% CI, 0.70 to 0.85). Insulin, SU, meglitinide, and AGI failed to demonstrate any favorable effects on the secondary prevention of repeat PCI compared with patients not using those glucose lowering agents.
Patients treated with these agents were associated with increased risk of repeated PCI.

\section{Subgroup analyses stratified by BMI}

To analyze the preventive effects of different glucose-lowering agents on repeat revascularization with PCI, subgroup analyses were conducted by stratifying participants according to BMI (Table 3). The overall effects of different glucose-lowering agents on the risk of requiring repeat PCI were similar in the non-obese group. Patients in the obese group (BMI $\geq 25 \mathrm{~kg} / \mathrm{m}^{2}$ ) treated with TZD showed a decreased risk of requiring repeat PCI compared to those who were not treated with TZD (aHR, 0.77; 95\% CI, 0.63 to 0.95) (Supplemental Fig. S2). However, this favorable effect of TZD was not observed in the non-obese

Table 2. Risk of Repeat Revascularization with PCI According to Study Participants' Prescribed Glucose Lowering Agent during Follow-up

\begin{tabular}{|c|c|c|c|c|c|c|c|c|c|}
\hline & \multirow{2}{*}{ Number } & \multirow{2}{*}{$\begin{array}{c}\text { Repeat } \\
\text { PCI }\end{array}$} & \multirow{2}{*}{ Duration } & \multirow{2}{*}{$\begin{array}{c}\mathrm{IR}, / 1,000 \\
\text { PY }\end{array}$} & \multicolumn{5}{|c|}{$\operatorname{HR}(95 \% \mathrm{CI})$} \\
\hline & & & & & Model $1^{\mathrm{a}}$ & Model $2^{\mathrm{b}}$ & Model $3^{c}$ & Model $4^{\mathrm{d}}$ & Model $5^{\mathrm{e}}$ \\
\hline \multicolumn{10}{|c|}{ Insulin } \\
\hline No & 20,904 & 2,440 & $91,387.63$ & 26.7 & 1 (reference) & 1 (reference) & 1 (reference) & 1 (reference) & 1 (reference) \\
\hline Yes & 14,444 & 2,005 & $59,743.13$ & 33.6 & $1.25(1.18-1.33)$ & $1.24(1.16-1.31)$ & $1.23(1.15-1.30)$ & $1.21(1.14-1.29)$ & $1.15(1.08-1.22)$ \\
\hline \multicolumn{10}{|c|}{ Sulfonylurea } \\
\hline No & 14,342 & 1,700 & $60,835.35$ & 27.9 & 1 (reference) & 1 (reference) & 1 (reference) & 1 (reference) & 1 (reference) \\
\hline Yes & 21,006 & 2,745 & $90,295.41$ & 30.4 & $1.11(1.04-1.17)$ & $1.10(1.04-1.17)$ & $1.07(0.99-1.17)$ & $1.07(0.98-1.16)$ & $1.04(0.96-1.13)$ \\
\hline \multicolumn{10}{|c|}{ Metformin } \\
\hline No & 8,457 & 1,131 & $35,535.41$ & 31.8 & 1 (reference) & 1 (reference) & 1 (reference) & 1 (reference) & 1 (reference) \\
\hline Yes & 26,891 & 3,314 & $115,595.36$ & 28.7 & $0.90(0.84-0.96)$ & $0.90(0.85-0.97)$ & $0.74(0.68-0.82)$ & $0.75(0.68-0.82)$ & $0.77(0.70-0.85)$ \\
\hline \multicolumn{10}{|c|}{ Meglitinides } \\
\hline No & 33,722 & 4,175 & $144,400.00$ & 28.9 & 1 (reference) & 1 (reference) & 1 (reference) & 1 (reference) & 1 (reference) \\
\hline Yes & 1,626 & 270 & $6,730.76$ & 40.1 & $1.40(1.24-1.59)$ & $1.38(1.22-1.56)$ & $1.28(1.13-1.45)$ & $1.28(1.13-1.45)$ & $1.27(1.12-1.44)$ \\
\hline \multicolumn{10}{|l|}{ TZD } \\
\hline No & 33,552 & 4,232 & $143,495.12$ & 29.5 & 1 (reference) & 1 (reference) & 1 (reference) & 1 (reference) & 1 (reference) \\
\hline Yes & 1,796 & 213 & $7,635.64$ & 27.9 & $0.94(0.82-1.08)$ & $0.95(0.83-1.09)$ & $0.92(0.80-1.06)$ & $0.92(0.80-1.06)$ & $0.90(0.78-1.04)$ \\
\hline \multicolumn{10}{|c|}{ DPP4i } \\
\hline No & 22,849 & 2,924 & $100,675.21$ & 29.0 & 1 (reference) & 1 (reference) & 1 (reference) & 1 (reference) & 1 (reference) \\
\hline Yes & 12,499 & 1,521 & $50,455.56$ & 30.1 & $0.98(0.92-1.05)$ & $0.98(0.92-1.05)$ & $0.93(0.86-0.99)$ & $0.93(0.86-1.00)$ & $0.93(0.87-1.01)$ \\
\hline \multicolumn{10}{|l|}{ AGI } \\
\hline No & 29,851 & 3,590 & $126,461.18$ & 28.4 & 1 (reference) & 1 (reference) & 1 (reference) & 1 (reference) & 1 (reference) \\
\hline Yes & 5,497 & 855 & $24,669.59$ & 34.7 & $1.28(1.19-1.38)$ & $1.28(1.19-1.38)$ & $1.25(1.15-1.36)$ & $1.25(1.15-1.36)$ & $1.24(1.14-1.34)$ \\
\hline
\end{tabular}

The incidence rate is presented per 1,000 person-years (PY).

PCI, percutaneous coronary intervention; IR, incidence rate; HR, hazard ratio; CI, confidence interval; TZD, thiazolidinedione; DPP4i, dipeptidyl peptidase-4 inhibitor; AGI, alpha-glucosidase inhibitor.

${ }^{\mathrm{a}}$ Adjusted for age and sex; ${ }^{\mathrm{b}}$ Adjusted model $1+$ adjusted for household income, hypertension, dyslipidemia, current smoking, alcohol, regular exercise, and body mass index; ${ }^{\mathrm{C}}$ Adjusted model $2+$ adjusted for use of insulin, number of oral antidiabetic drugs; ${ }^{\mathrm{d}}$ Adjusted model $3+$ adjusted for fasting blood glucose; ${ }^{\mathrm{e}}$ Adjusted model $4+$ adjusted for duration of diabetes. 


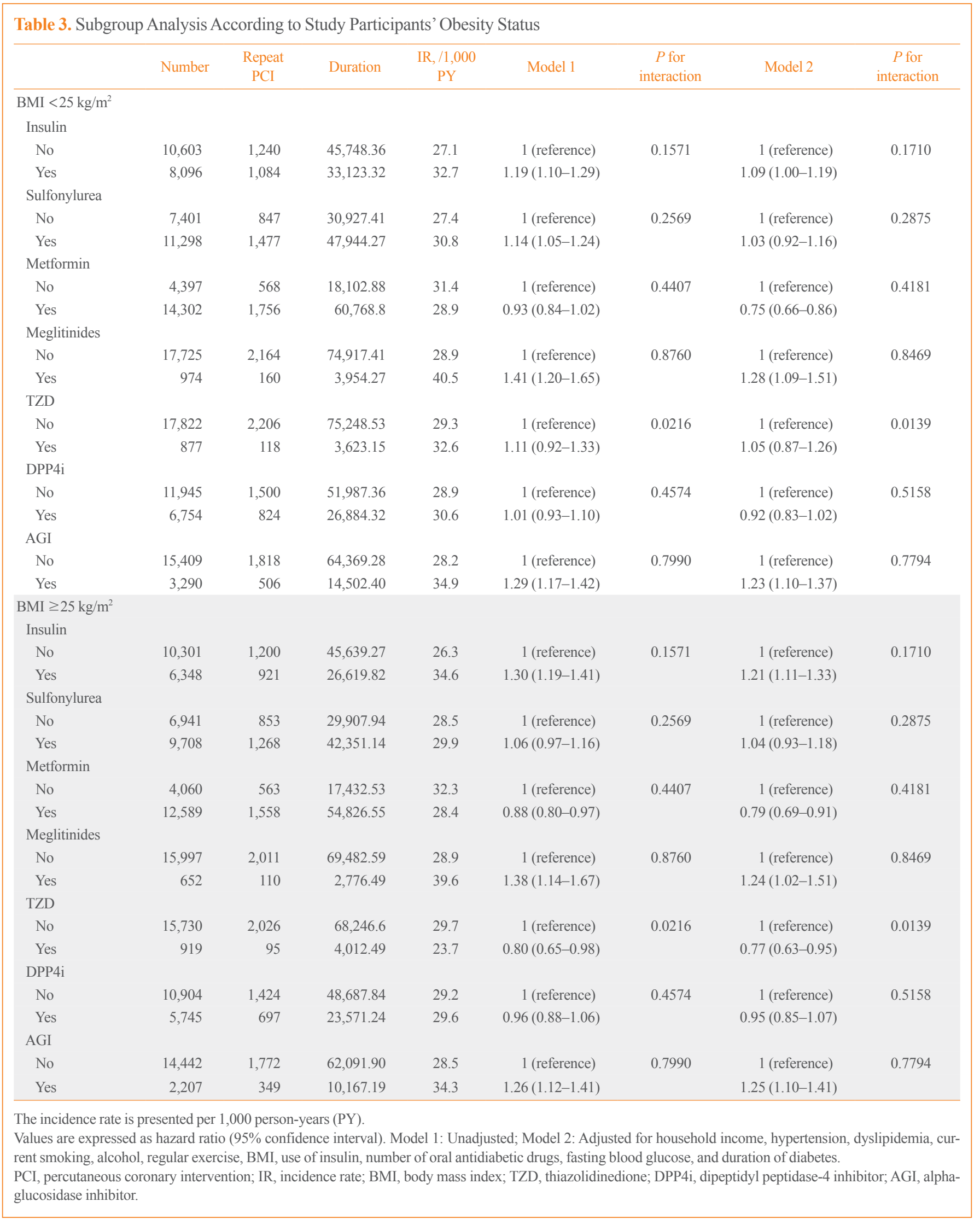




\begin{tabular}{|c|c|c|c|c|c|c|c|c|}
\hline & Number & Repeat PCI & Duration & $\begin{array}{c}\mathrm{IR}, / 1,000 \\
\text { PY }\end{array}$ & Model 1 & $\begin{array}{c}P \text { for } \\
\text { interaction }\end{array}$ & Model 2 & $\begin{array}{c}P \text { for } \\
\text { interaction }\end{array}$ \\
\hline \multicolumn{9}{|c|}{$\mathrm{BMI}<25 \mathrm{~kg} / \mathrm{m}^{2}$} \\
\hline \multicolumn{9}{|c|}{ No. of oral antidiabetic drugs } \\
\hline 0 & 2,398 & 263 & $9,956.05$ & 26.4 & 1 (reference) & \multirow[t]{4}{*}{0.0944} & 1 (reference) & \multirow[t]{4}{*}{0.0848} \\
\hline 1 & 3,461 & 397 & $14,770.92$ & 26.9 & $1.03(0.88-1.20)$ & & $1.02(0.88-1.20)$ & \\
\hline 2 & 6,180 & 791 & $26,499.35$ & 29.85 & $1.15(1.00-1.32)$ & & $1.13(0.98-1.30)$ & \\
\hline$\geq 3$ & 6,660 & 873 & $27,645.37$ & 31.6 & $1.19(1.04-1.37)$ & & $1.16(1.01-1.33)$ & \\
\hline \multicolumn{9}{|c|}{$\mathrm{BMI} \geq 25 \mathrm{~kg} / \mathrm{m}^{2}$} \\
\hline \multicolumn{9}{|c|}{ No. of oral antidiabetic drugs } \\
\hline 0 & 2,325 & 299 & $9,973.99$ & 30.0 & 1 (reference) & \multirow[t]{4}{*}{0.0944} & 1 (reference) & \multirow[t]{4}{*}{0.0848} \\
\hline 1 & 3,277 & 419 & $14,295.87$ & 29.3 & $0.98(0.85-1.14)$ & & $0.97(0.83-1.12)$ & \\
\hline 2 & 5,739 & 699 & $25,450.78$ & 27.5 & $0.93(0.81-1.06)$ & & $0.90(0.78-1.03)$ & \\
\hline$\geq 3$ & 5,308 & 704 & $22,538.44$ & 31.2 & $1.03(0.90-1.18)$ & & $0.98(0.85-1.12)$ & \\
\hline
\end{tabular}

group. Non-obese individuals who treated with multiple antidiabetic drugs showed a tendency of higher risk of repeat revascularization, without achieving statistical significance. In obese individuals, we did not observe significant association between the number of oral antidiabetic drugs and the risk of repeat revascularization (Table 4).

\section{Sensitivity analyses}

To minimize the bias, we performed sensitivity analysis in new users after excluding individuals with previous records of any glucose lowering agents before the index date of PCI (Supplemental Table S1). In patients who are newly diagnosed with T2DM, we observed a tendency of lower risk of repeat revascularization in patients treated with insulin, metformin, TZD and DPP-4 inhibitors without achieving statistical significance.

Regarding the changes of medication in individuals, we selected individuals who had not prescribed their previous oral antidiabetic medications at all after the index date of PCI and performed sensitivity analysis in those who have changed their oral glucose lowering agents after the index date of PCI (Supplemental Table S2). In this analysis, subjects treated with metformin, TZD and DPP-4 inhibitors after their index date of PCI had reduced risk of repeat PCI after adjusting for confounding variables including fasting blood glucose and duration of diabetes (aHR, 0.79, 0.73, and 0.87, respectively).

\section{DISCUSSION}

Several studies have shown that poor glycemic control after PCI might lead to worse clinical outcomes, including cardiac death, MI, restenosis after PCI, and stroke [12,13]. Hwang et al. [14] reported that good glycemic control after PCI was associated with a reduced rate of major adverse cardiac events, including repeat revascularization. Therefore, patients with T2DM need a strategy for glycemic control after PCI, which will be beneficial for preventing repeat revascularization. However, the preventive effect of glucose-lowering agents on restenosis after PCI in patients with T2DM using real-world data has not yet been reported.

In our study, we observed beneficial effects of metformin and DPP-4 inhibitors on reducing the risk of requiring revascularization after 4.3 years of follow-up. However, after adding the duration of diabetes in the final model, we observed lower risk of repeat revascularization in patients treated with metformin. Our results suggest that the duration of diabetes is an important confounder contributing to the risk of repeat revascularization.

Previous studies have suggested that metformin has a preventive effect on CAD in patients with T2DM [15]. The use of metformin in patients with diabetes undergoing coronary interventions seems to have beneficial effects on $\mathrm{CV}$ outcomes because it acts as an insulin sensitizer [16].

To date, there have been five CVOTs with DPP-4 inhibitors 
(The Saxagliptin Assessment of Vascular Outcomes Recorded in Patients with Diabetes Mellitus-Thrombolysis in Myocardial Infarction 53 [SAVOR-TIMI-53] trial, The Examination of Cardiovascular Outcomes with Alogliptin Versus Standard of Care [EXAMINE], The Trial Evaluating Cardiovascular Outcomes With Sitagliptin [TECOS], The Cardiovascular Outcome Study of Linagliptin Versus Glimepiride in Patients With Type 2 Diabetes [CAROLINA], and The Cardiovascular and Renal Microvascular Outcome Study With Linagliptin in Patients with Type 2 Diabetes Mellitus [CARMELINA]). Most of these trials demonstrated non-inferiority in terms of CV safety and its neutral effect on major CV events in patients with T2DM and established CVD [17]. We observed favorable effects of DPP-4 inhibitors on secondary prevention especially among patients who had undergone PCI and changed their medication, which is different from the results of previous CVOTs. These different results may be due to ethnic differences in pharmacodynamic responses [18]. Our results are in line with those of previous studies conducted in an Asian population. A case-control study conducted in Taiwan suggested the mortality benefits of DPP-4 inhibitors in patients with diabetes after the first acute myocardial infarction (AMI) [19]. Several hypotheses have been proposed to explain the benefits of DPP-4 inhibitors in patients with established CAD. First, DPP-4 inhibitors may alleviate the systemic proinflammatory state and reduce endothelial inflammation [20]. Furthermore, DPP-4 inhibitors may rescue mitochondrial dysfunction, which is associated with ischemia/reperfusion injury [21]. DPP-4 inhibitors may also inhibit the proliferation of vascular smooth muscle cells (VSMCs). In another study conducted in Japanese patients with multiple CV risk factors, DPP-4 inhibitors were shown to decrease blood pressure and improve albuminuria [22]. A systematic review and meta-analysis on the efficacy of DPP-4 inhibitors has proposed a better glucose-lowering efficacy in Asians than in other ethnic groups $[23,24]$. Future studies are required to explain these mechanisms, and ethnic-specific guidelines should be presented.

In subgroup analyses stratifying patients according to BMI, we observed a reduced risk of requiring repeat revascularization in obese patients treated with TZD. The secondary prevention effect of TZD on AMI was already confirmed in a PROactive study [8]. In high-risk patients with T2DM and previous MI, pioglitazone significantly reduced the occurrence of fatal and nonfatal MI and acute coronary syndrome [25]. This effect of pioglitazone could be partially explained by the mechanism of action of TZD. TZDs are agonists for the peroxisome proliferator-activated receptor- $\gamma$ (PPAR- $\gamma$ ), a nuclear receptor that is highly expressed in fat tissue [26]. As TZDs can regulate lipid pathways via adipocytes, their effects in obese patients may differ from those observed in non-obese patients. Moreover, researchers have established the presence of PPAR- $\gamma$ in human endothelial cells, vascular smooth muscle cells, monocytes, and human arterial lesions [27]. The activation of PPAR- $\gamma$ in vascular cells inhibits proliferation and migration of VSMCs [28]. Some studies suggest that TZDs may reduce neointimal tissue proliferation after stent implantation in patients with diabetes $[29,30]$. These findings suggest that TZDs have potential antiatherogenic effects. Further studies are required to confirm these findings.

We observed increased risk of repeated PCI among patients treated with insulin, SU, meglitinide and AGI. Coleman et al. [31] suggested no overall impact of AGI on CV outcomes in a meta-analysis and concluded that AGIs would rather not be indicated for $\mathrm{CV}$ secondary prevention. There are ongoing debates regarding the use of insulin in patients at high risk of CAD. In the Bypass Angioplasty Revascularization Investigation in Type 2 Diabetes (BARI 2D) trials, the risk of repeat revascularization did not differ by randomized treatment with insulin sensitizers versus insulin providers. The 5-year cumulative rate of repeat revascularizations was 0.32 for insulin sensitizers versus 0.34 for insulin providers $(P=0.08)$ [32]. However, in the Future Revascularization Evaluation in Patients With Diabetes Mellitus: Optimal Management of Multivessel Disease (FREEDOM) trial reported that insulin-treated diabetic patients have worse CV outcomes (comprises all-cause mortality, stroke, MI) regardless of the treatment arm [33]. Several observational studies also have found insulin users have a higher risk of $\mathrm{CV}$ and all-cause mortality compared to oral glucose lowering agents [34-36]. Our findings are consistent with the results of these previous cohort studies. Individuals treated with insulin in observational studies may have had poorer glycemic control, are more likely to have end-stage renal disease or other micro/ macrovascular diabetes complications. Moreover, insulin and insulin secretagogues (SU, meglitinide) are known to have weight gain effects and risk of hypoglycemia, might be associated with increases in MACE [37,38]. In our study, patients treated with insulin, meglitinides and AGIs had relatively long duration of diabetes. Although we included duration of diabetes as an adjustment variable in Cox proportional hazards model, a longer diabetes duration may associated with older age, which might have residual confounding effects.

Our study has potential limitations that should be considered when interpreting the results. First, detailed information about 
PCI, such as the type of DES, was not available in the NHIS database. Second, we did not consider the effects of other medications (i.e., antihypertensive drugs, lipid lowering agents, and anticoagulants), which might have potential effects on the development of $\mathrm{CV}$ complications. We also could not adjust for changes in fasting blood glucose, blood pressure or lipid profiles in individuals during follow-up period, because we could not obtain time-varying confounders in our dataset. Furthermore, we could not obtain information regarding procedures, such as the number of target vessels for PCI. We did not know glycated hemoglobin levels or the exact duration of diabetes mellitus, which might have potential effects on the clinical outcomes. The NHIS database has been established since January 2002. Therefore, it is possible that individuals who had history of T2DM before 2002 could be underestimated their duration of diabetes. Since we used limited data from NHIS not including HbA1c or presence of other diabetes complications, we tried our best to minimize the effects of the confounders through adjusting for insulin use and number of oral agents. The use of multiple antidiabetic agents or insulin, has been found to be an indicator of worse glycemic control in retrospective studies using administrative data [39]. We were unable to consider compliance during the follow-up period and the duration of use for each anti-diabetic agents. Regarding the changes of medication in individuals, sensitivity analysis consistently showed that individuals treated with metformin, TZD and DPP-4 inhibitors after their index date of PCI tend to have lower risk of repeat PCI. However, we could not obtain the detailed information about previously prescribed drugs and the exact combination of antidiabetic agents. Another limitation is the exclusion of SGLT-2 inhibitors and GLP-1RA, which has been proven to be effective for secondary prevention in T2DM patients with established ischemic heart disease. The GLP-1RA was introduced in Korea in 2008, and an SGLT-2 inhibitor was introduced in Korea at the end of 2013. After its introduction, GLP-1RA was available only in November 2010 with strict conditions for health insurance coverage [40]. These novel agents have proven safety and CV benefits beyond glucose control [41]; however, reimbursement restriction is a hurdle for prescription [42]. Therefore, owing to the low prescription rate during the study period, we did not include SGLT-2 inhibitors and GLP-1RAs in the analysis. Lastly, generalization of our results may be limited because of the single ethnicity of participants in this study.

Despite these limitations, our study had several strengths. First, this is a nationwide, population-based cohort study that investigated the effects of different glucose-lowering agents on the risk of requiring repeat PCI. Because of the difficulties encountered when conducting randomized controlled trials, no study has compared glucose-lowering agents and their preventive effects on repeat PCI, especially in patients who underwent PCI. Additional studies involving other ethnic groups with detailed information on coronary revascularization are required to clarify the preventive effects of drugs in these high-risk patients.

Among diabetic patients who underwent PCI, treatment with metformin seems to have an association with a reduced risk of requiring a repeat revascularization. When the risk of requiring repeat revascularization was analyzed in two groups according to the BMI, obese individuals treated with TZD were associated with a reduced risk of requiring repeat revascularization. These results suggest that physicians should choose appropriate glucose-lowering agents for the secondary prevention of CAD.

\section{CONFLICTS OF INTEREST}

ChongKunDang Pharmaceutical Company, Seoul, Republic of Korea provided financial support for this study.

\section{ACKNOWLEDGMENTS}

This work was supported by EnM Research Award 2018 of Korean Endocrine Society. The authors acknowledge the efforts of the Department of R\&D Management at Kangbuk Samsung Hospital, Korea, for editing the figures and tables. The authors would like to thank the National Health Insurance Service for their cooperation.

\section{AUTHOR CONTRIBUTIONS}

Conception or design: I.J. Acquisition, analysis, or interpretation of data: H.K., S.E.P., K.D.H., Y.G.P. Drafting the work or revising: I.J., H.K., S.E.P., E.J.R., W.Y.L. Final approval of the manuscript: I.J., H.K., S.E.P., K.D.H., Y.G.P., E.J.R., W.Y.L.

\section{ORCID}

Inha Jung https://orcid.org/0000-0001-8561-8544

Eun-Jung Rhee https://orcid.org/0000-0002-6108-7758

Won-Young Lee https://orcid.org/0000-0002-1082-7592

\section{REFERENCES}

1. Kannel WB, McGee DL. Diabetes and cardiovascular dis- 
ease: the Framingham study. JAMA 1979;241:2035-8.

2. Haffner SM, Lehto S, Ronnemaa T, Pyorala K, Laakso M. Mortality from coronary heart disease in subjects with type 2 diabetes and in nondiabetic subjects with and without prior myocardial infarction. N Engl J Med 1998;339:229-34.

3. Riche DM, Valderrama R, Henyan NN. Thiazolidinediones and risk of repeat target vessel revascularization following percutaneous coronary intervention: a meta-analysis. Diabetes Care 2007;30:384-8.

4. Center for Drug Evaluation and Research (CDER). Guidance for industry: diabetes mellitus-evaluating cardiovascular risk in new antidiabetic therapies to treat type 2 diabetes [Internet]. Silver Spring: US Food and Drug Administration; 2008 [cited 2021 Sep 3]. Available from: https://www.fda. gov/media/71297/download.

5. Hinnen D, Kruger DF. Cardiovascular risks in type 2 diabetes and the interpretation of cardiovascular outcome trials. Diabetes Metab Syndr Obes 2019;12:447-55.

6. Toh S, Hampp C, Reichman ME, Graham DJ, Balakrishnan S, Pucino F, et al. Risk for hospitalized heart failure among new users of saxagliptin, sitagliptin, and other antihyperglycemic drugs: a retrospective cohort study. Ann Intern Med 2016; 164:705-14.

7. Marx N, Rosenstock J, Kahn SE, Zinman B, Kastelein JJ, Lachin JM, et al. Design and baseline characteristics of the CARdiovascular outcome trial of LINAgliptin versus glimepiride in type 2 diabetes (CAROLINA(R)). Diab Vasc Dis Res 2015; 12:164-74.

8. Dormandy JA, Charbonnel B, Eckland DJ, Erdmann E, Massi-Benedetti M, Moules IK, et al. Secondary prevention of macrovascular events in patients with type 2 diabetes in the PROactive Study (PROspective pioglitAzone Clinical Trial In macroVascular Events): a randomized controlled trial. Lancet 2005;366:1279-89.

9. Nissen SE, Wolski K. Effect of rosiglitazone on the risk of myocardial infarction and death from cardiovascular causes. N Engl J Med 2007;356:2457-71.

10. Noh J. The diabetes epidemic in Korea. Endocrinol Metab (Seoul) 2016;31:349-53.

11. Hampp C, Borders-Hemphill V, Moeny DG, Wysowski DK. Use of antidiabetic drugs in the U.S., 2003-2012. Diabetes Care 2014;37:1367-74.

12. Asakura Y, Suzuki M, Nonogi H, Haze K, Sato A, Inada H, et al. Restenosis after percutaneous transluminal coronary angioplasty in patients with non-insulin-dependent diabetes mellitus (NIDDM). J Cardiovasc Risk 1998;5:331-4.
13. Chung JW, Park YS, Seo JE, Son Y, Oh CW, Lee CH, et al. Clinical impact of dysglycemia in patients with an acute myocardial infarction. Diabetes Metab J 2021;45:270-4.

14. Hwang JK, Lee SH, Song YB, Ahn J, Carriere K, Jang MJ, et al. Glycemic control status after percutaneous coronary intervention and long-term clinical outcomes in patients with type 2 diabetes mellitus. Circ Cardiovasc Interv 2017; 10:e004157.

15. Scheen AJ, Paquot N. Metformin revisited: a critical review of the benefit-risk balance in at-risk patients with type 2 diabetes. Diabetes Metab 2013;39:179-90.

16. Kao J, Tobis J, McClelland RL, Heaton MR, Davis BR, Holmes DR Jr, et al. Relation of metformin treatment to clinical events in diabetic patients undergoing percutaneous intervention. Am J Cardiol 2004;93:1347-50.

17. Gallwitz B. Clinical use of DPP-4 inhibitors. Front Endocrinol (Lausanne) 2019;10:389.

18. Chaturvedi N, Eastwood S. Prescribing by ethnicity: (im) precision medicine? Diabetes Care 2020;43:1687-9.

19. Wang MT, Lin SC, Tang PL, Hung WT, Cheng CC, Yang JS, et al. The impact of DPP-4 inhibitors on long-term survival among diabetic patients after first acute myocardial infarction. Cardiovasc Diabetol 2017;16:89.

20. Connelly KA, Zhang Y, Advani A, Advani SL, Thai K, Yuen DA, et al. DPP-4 inhibition attenuates cardiac dysfunction and adverse remodeling following myocardial infarction in rats with experimental diabetes. Cardiovasc Ther 2013;31: 259-67.

21. Kalogeris T, Bao Y, Korthuis RJ. Mitochondrial reactive oxygen species: a double edged sword in ischemia/reperfusion vs preconditioning. Redox Biol 2014;2:702-14.

22. Nakamura T, Iwanaga Y, Miyaji Y, Nohara R, Ishimura T, Miyazaki S, et al. Cardiovascular efficacy of sitagliptin in patients with diabetes at high risk of cardiovascular disease: a 12-month follow-up. Cardiovasc Diabetol 2016;15:54.

23. Kim YG, Hahn S, Oh TJ, Kwak SH, Park KS, Cho YM. Differences in the glucose-lowering efficacy of dipeptidyl peptidase-4 inhibitors between Asians and non-Asians: a systematic review and meta-analysis. Diabetologia 2013;56: 696-708.

24. Cai Y, Zeng T, Wen Z, Chen L. Ethnic differences in efficacy and safety of alogliptin: a systematic review and metaanalysis. Diabetes Ther 2018;9:177-91.

25. Erdmann E, Dormandy JA, Charbonnel B, Massi-Benedetti M, Moules IK, Skene AM, et al. The effect of pioglitazone on recurrent myocardial infarction in 2,445 patients with 
type 2 diabetes and previous myocardial infarction: results from the PROactive (PROactive 05) Study. J Am Coll Cardiol 2007;49:1772-80.

26. Tordjman J, Chauvet G, Quette J, Beale EG, Forest C, Antoine B. Thiazolidinediones block fatty acid release by inducing glyceroneogenesis in fat cells. J Biol Chem 2003; 278:18785-90.

27. Law RE, Goetze S, Xi XP, Jackson S, Kawano Y, Demer L, et al. Expression and function of PPARgamma in rat and human vascular smooth muscle cells. Circulation 2000;101: 1311-8.

28. Law RE, Meehan WP, Xi XP, Graf K, Wuthrich DA, Coats W, et al. Troglitazone inhibits vascular smooth muscle cell growth and intimal hyperplasia. J Clin Invest 1996;98:1897905.

29. Takagi T, Yamamuro A, Tamita K, Yamabe K, Katayama M, Mizoguchi S, et al. Pioglitazone reduces neointimal tissue proliferation after coronary stent implantation in patients with type 2 diabetes mellitus: an intravascular ultrasound scanning study. Am Heart J 2003;146:E5.

30. Choi D, Kim SK, Choi SH, Ko YG, Ahn CW, Jang Y, et al. Preventative effects of rosiglitazone on restenosis after coronary stent implantation in patients with type 2 diabetes. Diabetes Care 2004;27:2654-60.

31. Coleman RL, Scott CA, Lang Z, Bethel MA, Tuomilehto J, Holman RR. Meta-analysis of the impact of alpha-glucosidase inhibitors on incident diabetes and cardiovascular outcomes. Cardiovasc Diabetol 2019;18:135.

32. Dagenais GR, Lu J, Faxon DP, Kent K, Lago RM, Lezama C, et al. Effects of optimal medical treatment with or without coronary revascularization on angina and subsequent revascularizations in patients with type 2 diabetes mellitus and stable ischemic heart disease. Circulation 2011;123:1492500 .

33. Dangas GD, Farkouh ME, Sleeper LA, Yang M, Schoos MM, Macaya C, et al. Long-term outcome of PCI versus
CABG in insulin and non-insulin-treated diabetic patients: results from the FREEDOM trial. J Am Coll Cardiol 2014; 64:1189-97.

34. Roumie CL, Greevy RA, Grijalva CG, Hung AM, Liu X, Murff HJ, et al. Association between intensification of metformin treatment with insulin vs sulfonylureas and cardiovascular events and all-cause mortality among patients with diabetes. JAMA 2014;311:2288-96.

35. Currie CJ, Poole CD, Evans M, Peters JR, Morgan CL. Mortality and other important diabetes-related outcomes with insulin vs other antihyperglycemic therapies in type 2 diabetes. J Clin Endocrinol Metab 2013;98:668-77.

36. Gamble JM, Simpson SH, Eurich DT, Majumdar SR, Johnson JA. Insulin use and increased risk of mortality in type 2 diabetes: a cohort study. Diabetes Obes Metab 2010;12:4753.

37. Bell DS, Patil HR, O'Keefe JH. Divergent effects of various diabetes drugs on cardiovascular prognosis. Rev Cardiovasc Med 2013;14:e107-22.

38. Hanefeld M, Frier BM, Pistrosch F. Hypoglycemia and cardiovascular risk: is there a major link? Diabetes Care 2016; 39 Suppl 2:S205-9.

39. Willey CJ, Andrade SE, Cohen J, Fuller JC, Gurwitz JH. Polypharmacy with oral antidiabetic agents: an indicator of poor glycemic control. Am J Manag Care 2006;12:435-40.

40. Ko SH, Han K, Lee YH, Noh J, Park CY, Kim DJ, et al. Past and current status of adult type 2 diabetes mellitus management in Korea: a National Health Insurance Service Database analysis. Diabetes Metab J 2018;42:93-100.

41. Schnell O, Ryden L, Standl E, Ceriello A; D\&CVD EASD Study Group. Updates on cardiovascular outcome trials in diabetes. Cardiovasc Diabetol 2017;16:128.

42. Kim JY, Kim SJ, Nam CM, Moon KT, Park EC. Changes in prescription pattern, pharmaceutical expenditure and quality of care after introduction of reimbursement restriction in diabetes in Korea. Eur J Public Health 2018;28:209-14. 